\title{
A Modified Technique to Facilitate Anastomosis in Choledochal Cyst
}

\author{
Sezen Özkısacık, Mesut Yazıcı, Harun Gürsoy.
}

Adnan Menderes Universitesi Cocuk Cerrahisi Anabilim Dali, Aydin,Turkey.

Email: \{sozkisacik, myazici, sozkisacik\}@adu.edu.tr, harungursoy56@gmail.com

Received September $10^{\text {th }}$, 2010; revised september $28^{\text {th }}$, 2010; accepted October $27^{\text {th }}, 2010$.

\begin{abstract}
The method of choice for the treatment of choledochal cysts in children is excision of the cyst and Roux-en-Y (RY) choledochoenterostomy. When the ratio of the diameter of the main hepatic ductus to that of the proximal RY jejunum is 1:2.5 or lower during choledochoenterostomy, end-to-end anastomosis is recommended. However, this method may cause a difference in diameters between the ends. Here we will present the technical difficulty we experienced due to the difference in diameters in end-to-end RY choledochoenterostomy and our modified anastomosis technique of anastomosis. This slight modification eliminated problems with anastomosis caused by a difference in the diameter of the jejunum and shortened operation time.
\end{abstract}

Keywords: Anastomosis, Choledoch, Cyst

\section{Introduction}

Choledochal cysts are rare. The method of choice for the treatment of choledochal cysts in children is excision of the cyst and Roux-en-Y (RY) choledochoenterostomy to avoid malignant change [1]. The operative management is difficult because of inflammatory changes and fibrosis. When the ratio of the diameter of the main hepatic duct to that of proxymal RY jejunum is 1:2.5 or lower during choledochoenterostomy, end-to-end anastomosis is recommended [1]. If end-to-side anastomosis is inevitable, it should be performed on the most distal end of the blind jejunal pouch possible [2]. As children grow up, blind jejunal pouch can get longer when end-to-side anastomosis is performed and this can cause bile stasis, which may result in bile stone formation in intrahepatic bile ducts, pancreatitis and cholangitis [1,3]. In addition, adhesions may occur between the blind pouch and small bowel segments and vascular structures of the RY leg compresses the duodenum, which in turn leads to duodenal obstruction [2]. End-to-end anastomosis prevents all above complications. Therefore, we preferred end-to-end anastomosis. However, this method may cause a difference in diameters between the ends.

Here we will present the technical difficulty we experienced due to the difference in diameters in end-to-end RY choledochoenterestomy and how we solved it.

\section{Case}

A nine-year-old girl with cerebral palsy presented to our clinic with multiple calculi in the gallbladder detected on abdominal USG (Ultrasonography) and CT (Computerised Tomography) performed upon her frequent restlessness. Direct abdominal x-ray taken when the patient was standing showed two air-fluid levels on the posterior upper quadrant and USG demonstrated a choledochal cyst containing multiple calculi. When MR (Magnetic Resonance) cholangiography confirmed that the patient had a choledochal cyst containing multiple stones, we decided to operate on the patient.

\section{Surgical Technique}

The patient underwent laparotomy through an incision made on the right subcostal region. On intraoperative USG and cholangiography, intrahepatic bile ducts were dilated and the diameter of the cyst was larger than $5 \mathrm{~cm}$. The gallbladder and the cyst were excised on $1 \mathrm{~cm}$ proximal to the point where the common bile duct opens into the dudenum and $1 \mathrm{~cm}$ distal to the point where the right and left hepatic ducts merged. End-to-end RY choledochojejunostomy was performed.

Before anastomosis, the diameter of the jejunum was compared with that of the duct and no considerable difference was observed. We planned to use full-thickness 


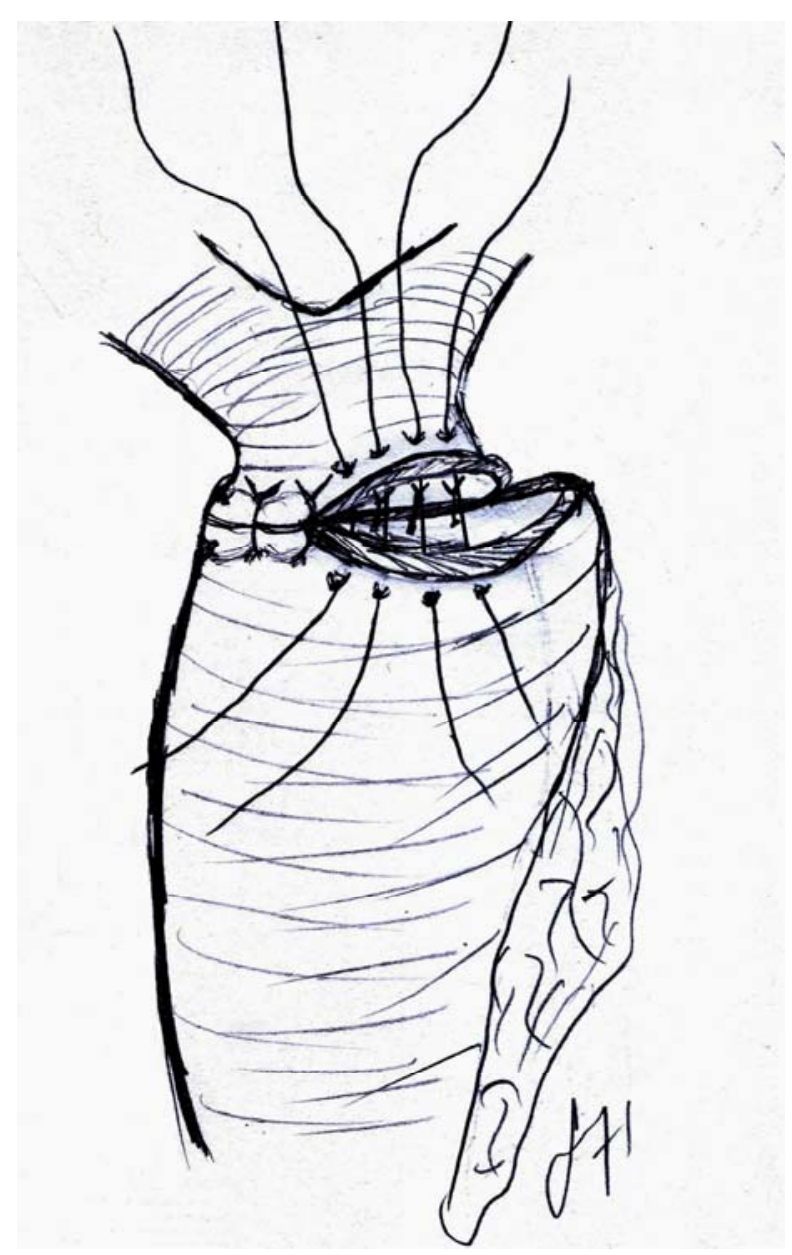

Figure 1. Difference in the diameter between the proximal aspect of the choledoch and jejunum ans and the jejunum entrance.

single sutures, to start anastomosis in the middle of the posterior wall, to proceed towards the anterior wall and to finish it in one corner of the wall. However, when we finished anastomosis of the back wall and started anastomosis of the anterior wall, we recognized a considerable difference in the diameter between the proximal aspect of the duct and jejunum ans and the jejunum entrance (Figure 1). It may be that the jejunum was more elastic than the duct. Therefore, we failed to accurately identify the ends to perform anastomosis and had to remove the sutures. We performed anastomosis again and put one sling suture in the corners and a whole thickness suture just in the middle of these sutures on the back walls. We put one more suture just between these sutures and sling sutures both in the right and the left sides. In addition, we put whole thickness anastomosis sutures between these sutures and sling sutures and between the two sutures put in the midline and completed anastomosis. Similarly, whole thickness single sutures were used

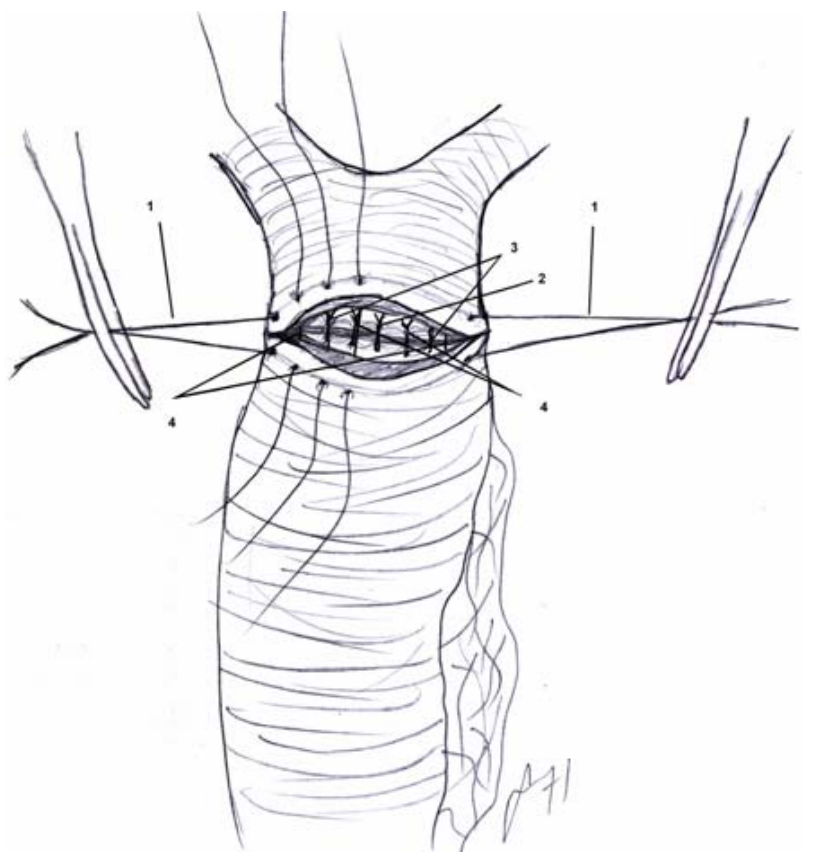

Figure 2 Anastomosis technique between jejunal ans and choledoch. (1: first suture; 2: second suture; 3: third suture; 4: fourth suture).

in the anterior wall and anastomosis was completed without any problems due to elasticity of the jejunum (Figure 2).

\section{Discussion}

In one case undergoing RY choledochojejunostomy, although there was no considerable difference in the diameter of the jejunum as a result of the anastomosis technique we used at first, later 'elasticity' of the jejunum increased, which caused a difference in the diameter of the jejunum, which in turn led us to modify the anastomosis techniques. This slight modification eliminated problems with anastomosis caused by a difference in the jejunum diameter and shortened operation time.

\section{REFERENCES}

[1] P. Puri and M. E. Höllwarth, Pediatric Surgery, 2006. pp. 371-386.

[2] A. Yamataka, H. Kobayashi, A. Shimotakahara, Y. Okada, T. Yanai, G. J. Lane, M. Urao and T. Miyano, "Recommendations for Preventing Complications Related to Roux-En-Y Hepatico-Jejunostomy Performed During Excision of Choledochal Cyst in Children," Journal of Pediatric Surgery, Vol. 38, 2003, pp. 1830-1832.

[3] R. Ohi, S. Yaoita, T. Kamiyama, M. Ibrahim, Y. Hayashi and T. Chiba, "Surgical Treatment of Congenital Dilatation of the Bile Duct with Special Reference to Late Complications After Total Excisional Operation,” Journal of Pediatric Surgery, Vol. 25, 1990, pp. 613-617. 